\title{
A NOTE ON CONDENSATIONS OF FUNCTION SPACES ONTO $\sigma$-COMPACT AND ANALYTIC SPACES
}

\author{
MIKOŁAJ KRUPSKI \\ (Communicated by Mirna Dzamonja)
}

\begin{abstract}
Modifying a construction of W. Marciszewski we prove (in ZFC) that there exists a subspace $X$ of the real line $\mathbb{R}$, such that the realcompact space $C_{p}(X)$ of continuous real-valued functions on $X$ with the pointwise convergence topology does not admit a continuous bijection onto a $\sigma$-compact space. This answers a question of Arhangel'skii.
\end{abstract}

\section{INTRODUCTION}

In this article, unless otherwise stated, by a space we mean a Hausdorff topological space. For a space $X$, we denote by $C_{p}(X)$ the space of all continuous real-valued functions on $X$ equipped with the pointwise convergence topology. It is well known that for a completely regular space $X$ the space $C_{p}(X)$ is almost never $\sigma$-compact. Namely, it is $\sigma$-compact if and only if the space $X$ is finite (see [11, 1.2.186]). Thus it is natural to ask which $C_{p}(X)$ spaces admit a weaker $\sigma$-compact (compact) topology, approximating the original one. This general problem posed by Arhangel'skii can be reformulated using a concept of condensation, i.e., continuous bijection: When is there a condensation of $C_{p}(X)$ onto a $\sigma$-compact (compact) space? (see, e.g., [7, Problem 5.1]).

For example, as was proved by Michalewski in $\left[8\right.$, the space $C_{p}(X)$ condenses onto a (metrizable) compactum whenever $X$ is a metrizable analytic space, i.e., $X$ is a continuous image of the space $\omega^{\omega}$ of the irrationals. Let us recall two problems posed by Arhangel'skii concerning the possible generalizations of the result mentioned above.

Problem 1.1. (Arhangel'skii, [2, Problem 4]) Suppose that $X$ is a separable metrizable space. Does $C_{p}(X)$ condense onto a $\sigma$-compact space?

Problem 1.2. (Arhangel'skii, [1, Problem 37]) Suppose that $C_{p}(X)$ is realcompact. Does $C_{p}(X)$ condense onto a $\sigma$-compact space?

Note that Problem 1.2, which was also asked in the recent book of Tkachuk 11, 4.10.1], is more general than Problem 1.1. Indeed, the space $C_{p}(X)$ is realcompact provided $X$ is separable (see [11, Problems 418 and 429]).

Received by the editors November 15, 2013.

2010 Mathematics Subject Classification. Primary 54C35, 54A10, 54D60.

Key words and phrases. Function space, pointwise convergence topology, $C_{p}(X)$ space, condensation, realcompact space.

The author was partially supported by the Polish National Science Center research grant UMO-2012/07/N/ST1/03525. 
A consistent negative answer to Problem 1.1 (and hence also to Problem 1.2) was given by Marciszewski in [6]. He constructed, assuming that $\mathfrak{d}=2^{\omega}$, a subspace $X$ of the real line $\mathbb{R}$ such that $C_{p}(X)$ does not condense onto a $\sigma$-compact space. However, the question if one can construct such a space without any additional set-theoretic assumptions remained open (see [7, page 363]).

In this short note we show how to modify the construction from [6] to make it work in ZFC. Thus the answer to both aforementioned problems is in the negative (see Theorem 3.1 and Corollary 3.2 below).

A significant part of the construction is the same as in [6] and therefore some details will be omitted. The results presented in the main text of $[6$ ] work for completely regular spaces. However, as was pointed out in [6, Remark], the construction of a space $X$ can be modified to the effect that $C_{p}(X)$ cannot be condensed onto a $\sigma$-compact Hausdorff space. It appears that as far as we are concerned with a condensation onto Tychonoff spaces, even the stronger result is true: the space $C_{p}(X)$ cannot be condensed onto any Tychonoff analytic space. We refer the interested reader to [7, Chapter 5], [10] and [11] for more information on condensations in $C_{p}$-theory and further references.

\section{AuXiliary Results}

For a space $X$ and its subset $D$, we denote by $C_{D}(X)$ the subspace $\{f \uparrow D: f \in$ $\left.C_{p}(X)\right\}$ of the space $C_{p}(D) \subseteq \mathbb{R}^{D}$. Note that the natural projection $\pi_{D}: \mathbb{R}^{X} \rightarrow \mathbb{R}^{D}$ condenses $C_{p}(X)$ onto $C_{D}(X)$, whenever $D$ is dense in $X$.

Proposition 2.1 given below was formulated in [6] without a proof (see [6, Remark $(2)])$. Since it is not completely straightforward we decided to enclose a short argument.

Proposition 2.1. Suppose that a space $X$ is a countable union of metrizable compacta. Then there exists an injective Borel map of $X$ onto a metrizable $\sigma$-compact space.

Proof. Since a finite union of metrizable compacta is a metrizable compactum we have $X=\bigcup_{n \in \omega} K_{n}$, where $K_{0}=\emptyset, K_{n} \subseteq K_{n+1}$ and each $K_{n}$ is metrizable compact. For $n \in \omega$, let $i_{n}: K_{n} \rightarrow[0,1]^{\omega}$ be a homeomorphic embedding. Let $f_{n}: X \rightarrow[0,1]^{\omega}$ be a Borel extension of $i_{n}$ taking $X \backslash K_{n}$ onto an arbitrary point. The diagonal map $F=\triangle_{n \in \omega} f_{n}: X \rightarrow\left([0,1]^{\omega}\right)^{\omega}$ is a Borel injection (every two points in $X$ belong to some $K_{n}$ and $f_{n}$ is injective on $\left.K_{n}\right)$. We shall prove that $F(X)$ is $\sigma$-compact. To this end it suffices to show that for each $n \in \omega$, the map $F$ is continuous on the $\sigma$-compact set $K_{n+1} \backslash K_{n}$. For $n=0$ this follows from the fact that each $f_{n}$ is continuous on $K_{n}$ and $K_{1} \subseteq K_{n}$, for $n \geqslant 1$. Let $n \geqslant 1$. For $m \geqslant n+1$ the map $f_{m}$ is continuous on $K_{n+1} \backslash K_{n} \subseteq K_{m}$ and for $m<n+1$ the map $f_{m}$ is continuous on $K_{n+1} \backslash K_{n}$ being constant (we assumed that $f_{m}$ maps $X \backslash K_{m}$ onto a point).

Proposition 2.2 ([6, Remark (3)]). Let E be a countable dense subset of a separable metrizable space $X$ and let $\psi: C_{p}(X) \rightarrow Y$ be continuous, where $Y$ is an arbitrary second countable space. Then $\psi$ has the following factorization property: There is a countable set $D \subseteq X$ containing $E$ and a continuous map $\xi: C_{D}(X) \rightarrow Y$ such that $\psi=\xi \circ\left(\pi_{D}\left\lceil C_{p}(X)\right)\right.$.

Proof. Let $\left\{U_{n}\right\}_{n \in \omega}$ be a countable base for $Y$. Since the space $X$ is separable metrizable it has a countable network. It follows that $C_{p}(X)$ also has a countable 
network and hence it is hereditarily Lindelöf. Thus, each open set in $C_{p}(X)$ is a countable union of basic open sets.

For each $n \in \omega$, we have $\psi^{-1}\left(U_{n}\right)=\bigcup_{m \in \omega}\left[A_{m}^{n}, V_{m}^{n}\right]$, where $\left[A_{m}^{n}, V_{m}^{n}\right]$ is a basic open set in $C_{p}(X)$ given by a finite set $A_{m}^{n} \subseteq X$ and an open set $V_{m}^{n} \subseteq \mathbb{R}$. It is easy to check that the set $D=E \cup \bigcup_{n, m \in \omega} A_{m}^{n}$ is as promised. The map $\xi$ is uniquely determined by $D$ because density of $D$ implies that $\pi_{D}$ is injective on $C_{p}(X)$.

The next proposition can be proved by a simple transfinite induction (cf. [9, the proof of 1.5.14]). It is a slight modification of the construction of the Bernstein set.

Proposition 2.3. There exists a set $\mathbb{K} \subseteq \mathbb{R}$ containing the rationals $\mathbb{Q}$ and such that both $\mathbb{K}$ and $\mathbb{R} \backslash \mathbb{K}$ intersect each copy of the Cantor set in $\mathbb{R}$.

Since in every Cantor set we can find continuum many pairwise disjoint Cantor sets, both $\mathbb{K}$ and $\mathbb{R} \backslash \mathbb{K}$ have cardinality $2^{\omega}$.

The next, easy proposition will be crucial in our construction.

Proposition 2.4. Let $\mathbb{K} \subseteq \mathbb{R}$ be the set given by Proposition 2.3. Suppose that $G \subseteq \mathbb{R}$ is a $G_{\delta}$ set containing $\mathbb{K}$. Then the set $\mathbb{R} \backslash G$ is countable.

Proof. Since $G$ is $G_{\delta}$, we have $\mathbb{R} \backslash G=\bigcup_{n \in \omega} F_{n}$, where each $F_{n}$ is closed. If $\mathbb{R} \backslash G$ were uncountable one of the sets $F_{n}$, being closed and uncountable, would contain a copy of the Cantor set. This however would contradict the property of $\mathbb{K}$.

\section{The COnstruction}

In this section we will prove the following:

Theorem 3.1. There is a space $X \subseteq \mathbb{R}$, containing the rationals $\mathbb{Q}$, such that $C_{p}(X)$ condenses neither onto a $\sigma$-compact (Hausdorff) space, nor onto an analytic Tychonoff space.

Proof. Let $\mathcal{F}$ be the following family of maps (cf. [6, Remark])

$\mathcal{F}=\left\{\varphi: B \rightarrow \mathbb{R}^{\omega}: B\right.$ is an analytic subset of $\mathbb{R}^{D}$, for some countable $D \subseteq \mathbb{R}$ with $\mathbb{Q} \subseteq D, \varphi$ is Borel\}.

The family $\mathcal{F}$ has cardinality $2^{\omega}$ and hence we can enumerate it as $\left\{\varphi_{\alpha}: B_{\alpha} \rightarrow\right.$ $\left.\mathbb{R}^{\omega}: \alpha<2^{\omega}\right\}$ (repetitions allowed) in such a way that $B_{\alpha}$ is a subset of $\mathbb{R}^{D_{\alpha}}$. Let $\mathbb{K} \subseteq \mathbb{R}$ be the set given by Proposition 2.3 .

In general, we are going to repeat the construction from [6]. The only change we are going to make is the starting point: We will use the set $\mathbb{K}$ instead of $\mathbb{Q}$. This simple idea allows us to drop the set-theoretic assumption $\mathfrak{d}=2^{\omega}$ required in 6 ] (cf. Remark 3.4 at the end of the paper). By induction we choose points $x_{\alpha}, y_{\alpha} \in \mathbb{R} \backslash \mathbb{K}$, $G_{\delta^{-}}$subsets $A_{\alpha}$ of $\mathbb{R}$ containing $\mathbb{K}$ and continuous functions $f_{\alpha}, g_{\alpha}: A_{\alpha} \rightarrow \mathbb{R}$ such that the following conditions are satisfied (we put $X_{\alpha}=\mathbb{K} \cup\left\{x_{\beta}: \beta<\alpha\right\}$ ).

(i) $x_{\beta} \neq x_{\alpha}$, for $\beta<\alpha$,

(ii) $\left(X_{\alpha} \cup\left\{x_{\alpha}\right\}\right) \cap\left\{y_{\beta}: \beta \leqslant \alpha\right\}=\emptyset$,

(iii) $x_{\alpha} \in(\mathbb{R} \backslash \mathbb{K}) \cap\left(\bigcap_{\beta \leqslant \alpha} A_{\beta}\right)$,

(iv) if $D_{\alpha} \backslash X_{\alpha} \neq \emptyset$, then $y_{\alpha} \in D_{\alpha}$,

(v) if $D_{\alpha} \subseteq X_{\alpha}$ and $C_{D_{\alpha}}\left(X_{\alpha}\right) \backslash B_{\alpha} \neq \emptyset$, then $X_{\alpha} \subseteq A_{\alpha}$ and $f_{\alpha} \uparrow D_{\alpha} \notin B_{\alpha}$,

(vi) if $D_{\alpha} \subseteq X_{\alpha}, C_{D_{\alpha}}\left(X_{\alpha}\right) \subseteq B_{\alpha}$ and $\varphi_{\alpha}\left\lceil C_{D_{\alpha}}\left(X_{\alpha}\right)\right.$ is not injective, then $X_{\alpha} \subseteq A_{\alpha}, f_{\alpha} \neq g_{\alpha}$ and $\varphi\left(f_{\alpha}\left\lceil D_{\alpha}\right)=\varphi\left(g_{\alpha}\left\lceil D_{\alpha}\right)\right.\right.$. 
Note that for $\alpha<2^{\omega}$ the set $(\mathbb{R} \backslash \mathbb{K}) \cap\left(\bigcap_{\beta \leqslant \alpha} A_{\beta}\right)$ has cardinality $2^{\omega}$. Indeed, for each $\beta \leqslant \alpha$ the set $A_{\beta}$ is a $G_{\delta}$-subset of $\mathbb{R}$ containing $\mathbb{K}$ and hence Proposition 2.4 implies that $\left|\left(\bigcap_{\beta \leqslant \alpha} A_{\beta}\right)^{c}\right|<2^{\omega}$. Moreover, as we have already observed, the set $\mathbb{R} \backslash \mathbb{K}$ has cardinality $2^{\omega}$.

Now, the inductive step can be made by considering the same four cases (corresponding to conditions (iv)-(vi)) as in [6].

Put $X=\mathbb{K} \cup\left\{x_{\alpha}: \alpha<2^{\omega}\right\}$. We need to show that there is no condensation from $C_{p}(X)$ onto a space $M$ being $\sigma$-compact or Tychonoff and analytic. The argument given in [6] works for $M$ being Tychonoff and $\sigma$-compact. If $M$ is just Hausdorff, the proof is a little bit different and for this reason we enclose a justification.

Let $M$ be $\sigma$-compact. Suppose that there is a condensation $\psi: C_{p}(X) \rightarrow M$. Since $X$ is separable metrizable, the space $C_{p}(X)$ has a countable network and so does $M$. Thus $M$ is a countable union of metrizable compacta. Since every space with a countable network condenses onto a space with a countable base [3. Ch. 2, Problem 149], without loss of generality we may assume that $M$ has a countable base. By Proposition 2.2, there exists a countable set $D \subseteq X$ containing $\mathbb{Q}$ and a map $\xi: C_{D}(X) \rightarrow M$ such that $\psi=\xi \circ\left(\pi_{D}\left\lceil C_{p}(X)\right)\right.$. Proposition 2.1 yields the existence of a metrizable $\sigma$-compact space $S$ and an injective Borel map $\eta: M \rightarrow S$ (in the case $M$ is an analytic Tychonoff space, by [11, Problem 156 (iii)] there exists a continuous map $\eta$ and a space $S$ with a countable base which is analytic as a continuous image of an analytic space $M$ ). As $S$ is metrizable, we can assume that $S \subseteq \mathbb{R}^{\omega}$. We take $\varphi^{\prime}=\eta \circ \xi: C_{D}(X) \rightarrow \mathbb{R}^{\omega}$. Using a theorem of Kuratowski (see $[4, \S 35 . \mathrm{VI}]$ ) we can find a Borel subset $B^{\prime}$ of $\mathbb{R}^{D}$ containing $C_{D}(X)$ and a Borel extension $\varphi^{\prime \prime}: B^{\prime} \rightarrow \mathbb{R}^{\omega}$ of a Borel injective map $\varphi^{\prime}$. From now on we proceed as in [6]. Take $B=\left(\varphi^{\prime \prime}\right)^{-1}(S)$ and $\varphi=\varphi^{\prime \prime} \uparrow B$. The set $B$ is analytic, being a Borel preimage of a $\sigma$-compact set (if $M$ were a Tychonoff analytic space, the set $B$ is a Borel preimage of an analytic set; hence, it is analytic) and $\varphi$ maps injectively $C_{D}(X)$ onto $S$. The map $\varphi$ belongs to $\mathcal{F}$ and thus there is $\alpha<2^{\omega}$ such that $\varphi=\varphi_{\alpha}, D=D_{\alpha}, B=B_{\alpha}$.

We consider the following four complementary cases:

Case 1. $D_{\alpha} \backslash X_{\alpha} \neq \emptyset$.

By (iv), $y_{\alpha} \in D_{\alpha}=D$ and $y_{\alpha} \notin X$ by (ii). Hence $D \backslash X \neq \emptyset$, a contradiction.

Case 2. $D_{\alpha} \subseteq X_{\alpha}$ and $C_{D_{\alpha}}\left(X_{\alpha}\right) \backslash B_{\alpha} \neq \emptyset$.

By (v), $X_{\alpha} \subseteq A_{\alpha}$ and by (iii) $x_{\beta} \in A_{\alpha}$ for $\beta \geqslant \alpha$, so $X \subseteq A_{\alpha}$ and $f_{\alpha}\lceil X \in$ $C_{p}(X)$. Condition (v) implies that $f_{\alpha} \uparrow D \in C_{D}(X) \backslash B$, a contradiction.

Case 3. $D_{\alpha} \subseteq X_{\alpha}, C_{D_{\alpha}}\left(X_{\alpha}\right) \subseteq B_{\alpha}$ and $\varphi_{\alpha} \uparrow C_{D_{\alpha}}\left(X_{\alpha}\right)$ is not injective.

Similarly as in Case 2, condition (vi) implies that $X \subseteq A_{\alpha}$ and $f_{\alpha} \uparrow X, g_{\alpha} \uparrow X \in$ $C_{p}(X)$. Then $f_{\alpha} \uparrow D, g_{\alpha} \uparrow D$ are distinct elements of $C_{D}(X)$ and $\varphi\left(f_{\alpha} \uparrow D\right)=$ $\varphi\left(g_{\alpha}\lceil D)\right.$, a contradiction.

Case 4. $D_{\alpha} \subseteq X_{\alpha}, C_{D_{\alpha}}\left(X_{\alpha}\right) \subseteq B_{\alpha}$ and $\varphi_{\alpha} \uparrow C_{D_{\alpha}}\left(X_{\alpha}\right)$ is injective.

By (i), $X_{\alpha}$ is a proper subset of $X$ and hence $C_{D}(X)$ is a proper subset of $C_{D}\left(X_{\alpha}\right)$ (see [6, Proposition 2.5]). Since $\varphi$ is injective on $C_{D}\left(X_{\alpha}\right)$ and $\varphi\left(C_{D}\left(X_{\alpha}\right)\right) \subseteq \varphi(B)=$ $S$, we have $S \backslash \varphi\left(C_{D}(X)\right) \neq \emptyset$, a contradiction. 
Theorem 3.1 gives a negative answer to Problem 1.1. As we mentioned in the Introduction it also immediately implies the following negative answer to Problem 1.2 .

Corollary 3.2. There is a space $C_{p}(X)$ which is realcompact and does not admit a condensation onto a $\sigma$-compact space.

Remark 3.3. It seems that the answer to Problem 1.2 has been known before. As R. Pol observed it is enough to consider $X=\omega \cup\{\infty\}$, where the points of $\omega$ are isolated and the neighborhoods of $\infty$ are given by an analytic non-Borel filter on $\omega$ (see [5, Theorem 4.1]). Then $C_{p}(X)$ is clearly realcompact and does not condense onto any Borel subset of a Polish space.

Remark 3.4. We feel that the reader deserves a better explanation why the proof of Theorem 3.1, although very similar to the construction from [6], does not require the set-theoretic assumption $\mathfrak{d}=2^{\omega}$ which was vital in [6]. Recall that $\mathfrak{d}=2^{\omega}$ is equivalent to the following statement:

(*) The intersection of less than continuum many $G_{\delta}$-subsets of $\mathbb{R}$ containing $\mathbb{Q}$ has cardinality $2^{\omega}$.

Now, if we start the construction of the space $X$ from $\mathbb{Q}$ and try to add points $x_{\alpha} \in \mathbb{R} \backslash \mathbb{Q}$ inductively, as it was done in [6], we will need (*) to fulfill conditions (i)(iii) of the construction. That is, we will need $(*)$ to make sure that the intersection $(\mathbb{R} \backslash \mathbb{Q}) \cap \bigcap_{\beta \leqslant \alpha} A_{\beta}$ is big enough (has cardinality $2^{\omega}$ ) to choose a point $x_{\alpha}$ belonging to it and distinct from points already chosen.

If we start the construction of $X$ from $\mathbb{K}$ (as it was done in the proof of Theorem 3.1), we force the intersection $(\mathbb{R} \backslash \mathbb{K}) \cap \bigcap_{\beta \leqslant \alpha} A_{\beta}$ to be big, simply by making sure that it contains the set $\mathbb{K}$. Thus we no longer need the statement $(*)$.

\section{ACKNOWLEDGMENT}

The author would like to thank Witold Marciszewski for discussions on the subject. The author is also indebted to Roman Pol for valuable suggestions and to Paweł Krupski and Grzegorz Plebanek for reading the preliminary version of the paper.

\section{REFERENCES}

[1] A. V. Arhangel'skii, $C_{p}$-Theory, in: Recent Progress in General Topology, M. Hušek and J. van Mill (eds.), Elsevier 1992, 1-56.

[2] A. V. Arhangel'skii, On condensations of $C_{p}$-spaces onto compacta, Proc. Amer. Math. Soc. 128 (2000), no. 6, 1881-1883 (electronic), DOI 10.1090/S0002-9939-00-05758-0. MR 1751998 (2001c:54002)

[3] A. V. Arkhangel'skiı̌ and V. I. Ponomarev, Fundamentals of general topology, Mathematics and its Applications, D. Reidel Publishing Co., Dordrecht, 1984. Problems and exercises; Translated from the Russian by V. K. Jain; With a foreword by P. Alexandroff [P. S. Aleksandrov]. MR785749 (87i:54001)

[4] K. Kuratowski, Topology. Vol. I, New edition, revised and augmented. Translated from the French by J. Jaworowski, Academic Press, New York-London; Państwowe Wydawnictwo Naukowe, Warsaw, 1966. MR0217751 (36 \#840)

[5] D. Lutzer, J. van Mill, and R. Pol, Descriptive complexity of function spaces, Trans. Amer. Math. Soc. 291 (1985), no. 1, 121-128, DOI 10.2307/1999898. MR797049 (87e:54046)

[6] Witold Marciszewski, A function space $C_{p}(X)$ without a condensation onto a $\sigma$-compact space, Proc. Amer. Math. Soc. 131 (2003), no. 6, 1965-1969 (electronic), DOI 10.1090/S00029939-02-06668-6. MR 1955287(2004a:54019) 
[7] Witold Marciszewski, Function spaces, Recent progress in general topology, II, North-Holland, Amsterdam, 2002, pp. 345-369, DOI 10.1016/B978-044450980-2/50013-3. MR1970004

[8] Henryk Michalewski, Condensations of projective sets onto compacta, Proc. Amer. Math. Soc. 131 (2003), no. 11, 3601-3606 (electronic), DOI 10.1090/S0002-9939-03-06882-5. MR1991774 (2004d:54015)

[9] Jan van Mill, The infinite-dimensional topology of function spaces, North-Holland Mathematical Library, vol. 64, North-Holland Publishing Co., Amsterdam, 2001. MR1851014 (2002h:57031)

[10] V. V. Tkachuk, Condensations of $C_{p}(X)$ onto $\sigma$-compact spaces, Appl. Gen. Topol. 10 (2009), no. 1, 39-48. MR2602601 (2011f:54028)

[11] Vladimir V. Tkachuk, $A C_{p}$-theory problem book, Problem Books in Mathematics, Springer, New York, 2011. Topological and function spaces. MR.3024898

Institute of Mathematics, Polish Academy of Sciences, Ul. Śniadeckich 8, 00-956 Warszawa, Poland

E-mail address: krupski@impan.pl 